\title{
Instrumentation designed to simulate the effects of prisms used in studies of visual rearrangement
}

\author{
KATHIE GOURLAY. JOHN W. GYR. STACEY WALTERS, and RICHMOND WILLEY \\ University of Michigan, Ann Arbor, Michigan 48104
}

\begin{abstract}
A system is described which simulates some of the visual rearrangements and changes in sensorimotor relations that occur when experimental subjects move their eyes while looking through a contact lens with a prism attached. The simulated system is more convenient than a system based on the use of a contact lens, and it is based on measures of eye movement that are important in research on the perceptual effects of visual rearrangement. The effects that occur are reviewed in this paper. We also show in detail how the artificial system, which has an eye-movement monitor interfaced with a computer and display, simulates the former effects. The data show that experimental subjects who experience visual rearrangement by simulated means manifest the same kind of perceptual adaptation produced in studies in which visual rearrangement is generated by means of a prism mounted on a contact lens.
\end{abstract}

The sistem. which simulates the effects produced by prisms. consists of an eye monitor. a PDP-9 computer. and a cathode ray tube (CRT). In order to describe system operation. it is necessary to review the effects produced by prisms as well as the experimental uses of prisnis reported in the literature.

\section{USE OF PRISMS IN PAST RESEARCH IN PERCEPTION}

Optic derices like prisns have been employed typically when an investigator wants to study perceptual adaptation to visual rearrangement. and thus wants to displace the apparent location of objects relative to an observer or to alter the shape of contours by making. for example, straight lines look curved or curred lines look straight. For a recent review of much of the literature. see Gyr (1972). Visual rearrangements such as the latter-when produced by a contact lens with a prism mounted on it-lead to unusual visual effects when subjects start to scan the rearranged contours by moving their eyes. For example. suppose subjects scan an apparently straight but physically curved contour in the usual way. i.e.. br means of a straight movement of the eye parallel to the contour: they expect to see a line which retains its orientation and is easily kept in focus. What the subjects see instead is a line that changes orientation as a function of their eve movements, and which therefore cannot be fixated by a straight eye novement. In fact. fixation can be maintained only by moving the eve in a parth similar to the true. curved. rather than the apparent. straight. shape of the object. Some perceptual adaptation theorists would say that subjects subjected to such experimental

Requests for reprints should be sent to John W. Gyr. Mental Health Research Institute. University of Michigan. Ann Arbor. Michigan 48104. conditions must learn a new sensorimotor correlation. Once learned. the theory suggests that perceptual adaptation will result. (In the case of perfect adaptation. subjects will call those contours curved that can be fixated under a curved movement of the eve. regardless of the shape the object projects on the retina: similarly, they will call those contours straight that can be fixated under a straight movement of the eve. Since. in these experiments, vision has been tampered with optically and motor movements have not. the theory holds that under conditions allowing voluntary movements during scanning. new visual signs $w$ ill become attached to already well-established motor movements.) In practice. no studies have been able to show perfect visual adaptation to curvature on the part of subjects. But evidence for partial visual adaptation has been presented in several research studies. Thus. Festinger. Burnham, Ono, and Bamber (1967) and Slotnick (1969), using contact lenses with prisms mounted on them, showed consistent trends in the direction of visual adaptation to optically produced shape distortions in three and in tive subjects. respectively. Probably because of the relatively low number of subjects. no statistical tests of adaptation were run.

\section{REASONS FOR SIMULATING EFFECTS OF PRISMS}

One of the obvious reasons for the small size of the experimental populations in the above studies was the cumbersome experimental procedure required. That is, at least one contact lens with attached prism had to be fitted to each subject. This circumstance in itself presents a strong argument for seeking other means of study: it led the present authors to become interested in a nonoptic methodology. ${ }^{1}$ Another important advantage of such simulation is that the required ere-morement measures can then be used as 
independent variables in the research. This is important if. as several theories hold. efference is centrally involved in the adaptation process. Since, in the conventional methodology, visual rearrangements are produced optically rather than artificially from a knowledge of the subjects' eve movements (see below). any measures of the latter have to be obtained separately and have typically been unavailable.

\section{DESCRIPTION OF THE SIMULATION OF A PRISM}

As indicated earlier, when subjects scan an apparently straight but physically curved contour while wearing contact lenses with prisms mounted on them. the contour changes its orientation as a function of eye movements and subjects thus cannot fixate the contour until ther learn to scan it by means of all eve path that is congruent with the contour's phisical rather than its apparent shape. A computer program. using information about the subject's eye position obtained with an eye-movement monitor and using the appropriate transformation equation. can produce on a CRT the same visual impressions of change in the orientation of a contour when scanned by the subjects. This. in fact. is the basic principle of the nonoptic methodology for producing visual rearrangement which will be described here. The particular instance studied, as was true for some of the studies by Festinger et al. and Slotnick, was that of subjects seeing an apparently straight but phisically curved contour on the CRT and being asked to scan it. A similar system, simulating the visual feedback received by subjects when they "draw" seen objects while wearing prism goggles, was designed by Gyr. Willey, and Gordon. 1972.

\section{Equipment}

The basic equipment consisted of a PDP-9 computer. a CRT display, and an eye-movement monitor.

The eye-movement monitor was a Biometrics Model 200. which is described in detail in the operator's manual available from the company. ${ }^{2}$ It employs a pair of silicon phototransistors and a gallium arsenide infrared light source in front of each eye. Either eye can be used for vertical or horizontal measurement, but one eve cannot be used for both dimensions. Changes in reflected light between the white sclera and the darker iris specify horizontal motion, and variations in total intensity resulting from eyelid movement specify vertical movement. The monitor is capable of measuring horizontal eye movements over a range of $\pm 20 \mathrm{deg}$. with resolution better than ${ }^{1}+$ deg. and vertical movements of $+10 \mathrm{deg}$ to -20 deg. with resolution better than $1 \mathrm{deg}$. The sensors are mounted on a pair of spectacle frames that are held in place with an elastic band around the back of the head. The position of each set of sensors relative to the eve can be varied in three dimensions. The sensors are connected by a cable to a table-top case containing the monitor's electronics. output terminals. meter, and variable controls for each eye. the meter monitors output from the left or right eye, but not both at the same time. The controis for each cre include: a gain control knob, a zero control knob, a crosstalk compensation control, a vertical/ horizontal mode selector switch. and a filter switch for increasing the instrument's time constant. The electrical inputs from the vertical and horizontal sensors are adjusted according to the setting of these control knobs. amplified to be in the range of $\pm 10 \mathrm{~V}$ dc, and then output to the analog-to-digital (A/D) converter.

The headrest initially consisted of a vertically adjustable frame on which could be mounted either a chinrest or a biteboard. The frame included a forchead rest, which surrounded the top of the head and constrained head movement via a pair of adjustable rings. Fine adjustments of the height and till of the chinrest or biteboard could be made independently of vertical frame adjustment.

A number of modifcations were made in the monitor and headrest. Some of these were judged to improve general efficiency and comfort; others were needed for the present. specific application.

(1) By means of small bolts and dental rubber bands mounted on the sensor frames, the position of the sensors relative to the eye in each dimension was made adjustable by tine turns of a bolt. This greatly increased ease and precision of adjustment, which previously had required that the sensors be slid manually and then held in place with setscrews.

(2) In pilot experiments, the spectacle frames became quite uncomfortable after a short time because of pressure on the bridge of the nose produced by the elastic headband. The discomfort was only partially alleviated by the addition of nose pads. Accordingly, the elastic band and earpieces of the spectacle frame were replaced by brackets that could be attached to the headband of a welding helmet (see Figure 1). The brackets and frame could be removed for initial fitting of the headband. They could then be reattached and adjusted so that the frame rested very lightly on the nose. This could be accomplished without discomfort from the headband itself.

(3) The small platforms on which the sensors and light sources were mounted were found to be too wide for effective horizontal adjustment, as they tended to hit the nose. Furthermore, in some cases. they were not deep enough to allow the sensors to come close enough to the eye. They were replaced by narrower and deeper platforms. The separation between the sensors and the light source was unchanged.

(4) The forehead rest was found to be inadequate in 
preventing head rotation unless painful pressure was applied. Also, it was too flexible to hold the head steady when the subject pressed forward against it. It was replaced by a narrow padded forehead rest attached to the main section of the headrest by means of a supplementary frame made of $1 / 2$-in. $(12.7-\mathrm{mm})$ aluminum rods (Flexaframe). For the present experiment, physical constraint of head rotation was judged to be unnecessary.

(5) The chinrest, used throughout the experiment, was covered with a polyurethane foam pad.

(6) In pilot work, it was found that, because of variations in head size, neither a fixed position of the forehead pad nor a fixed position of the chinrest guaranteed a constant vertical eye position. Hence, an attachment was made consisting of a panel with a narrow horizontal slit that could be moved up to a constant height on the supplementary frame. The subject rested against the forehead pad and moved his head up and down until a visual target was vertically centered in the slit. The chinrest was then adjusted to maintain this position. The panel with the slit could then be moved down out of the way.

(7) A large cardboard box, open at the back and containing a small aperture in front, was mounted on the headrest so as to surround the subject's head and restrict vision to a portion of the CRT screen. The box was vertically adjustable for precise positioning of the paerture and could be removed during sensor adjustments. The aperture contained a filter (one sheet of Wratten gelatin No. 47B and seven sheets of Vinylite transparent blue filter) which eliminated afterimages on the screen. With the room lights out, the subject could see only the illuminated forms on the CRT screen.

The computer system is part of the University of Michigan's Computing Center. It includes a PDP-9 with $16 \mathrm{k}$ of core memory and an extended arithmetic unit. The CRT is a Digital Equipment Corporation Type 339 programmed buffered display. The face of the CRT is a $24.2-\mathrm{cm}$-square area containing $1,024 \mathrm{x}$ 1,024 raster points. The 339 is a powerful display, capable of plotting both points and vectors at eight different intensity levels. The display is under programmable control by the PDP-9. The 339 has its own DPU (display processing unit), so that the display runs asynchronously with the CPU (central processing unit), i.e., the computer can be used for other operations simultaneous to the running of the display.

The $\mathrm{x}$ and $\mathrm{y}$ signals from the eye-movement monitor were input to the PDP-9 through an A/D convertermultiplexer (Digital Equipment Corporation Model AF01). Of the 64 possible analog input channels, only two were used to measure, respectively, the $x$ coordinate and the $y$ coordinate of the eye point-of-gaze. The 12-bit conversion ability of the AF01 is more than adequate to obtain precise conversion of the $2^{10}$ possible coordinate positions on

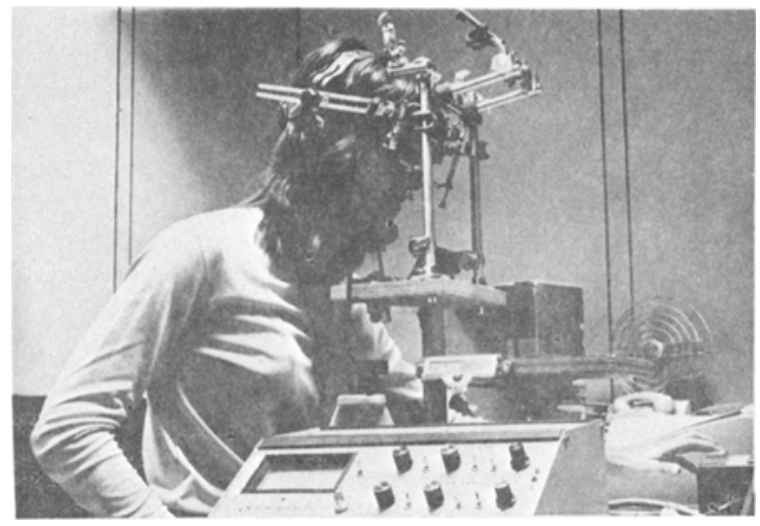

Figure 1. Subject wearing eye-movement monitor mounted on the head of a welding helmet.

the CRT. The AF01 takes 35 microsec to convert 12 bits of information.

The system includes as an input/output (I/O) device a small, movable push-button control box of 12 buttons. It was used by the subject or the experimenter to control the parameters of the image being displayed and to signal responses. Other $\mathrm{I} / \mathrm{O}$ devices include a paper-tape reader, paper-tape punch, and Teletype. The reader was used for inputting the computer programs and parameters. The punch and the Teletype were used to record data, while the Teletype was also used by the experimenter to set the initial parameters for a particular experimental condition.

\section{Programming}

The programs were written in assembler language. All the programs are under the control of a small, supervisor program which accepts commands from the Teletype and transfers control to the requested subprograms. Currently, there are five subprograms which perform: calibration, verification, monitoring, pre-/posttests, and prism simulation. The programs also make heavy use of the display routines of the SEL Executive System which were written for this computer and are supported by the University of Michigan Computing Center. The SEL Executive Systen uses most of the lower $8 \mathrm{~K}$ of the PDP-9, leaving $8 \mathrm{~K}$ for user routines, all of which were used for the present application.

Calibration. There is no intrinsic correspondence between the eye-movement monitor's output voltages and the point-of-gaze of the eye. It is therefore necessary, prior to each experiment, to obtain a transformation function that can be applied to the digitized inputs to convert them into the coordinate system of the CRT ( 0 to 1023).

It was initially assumed that the transformation was linear, so the calibration program was written to 


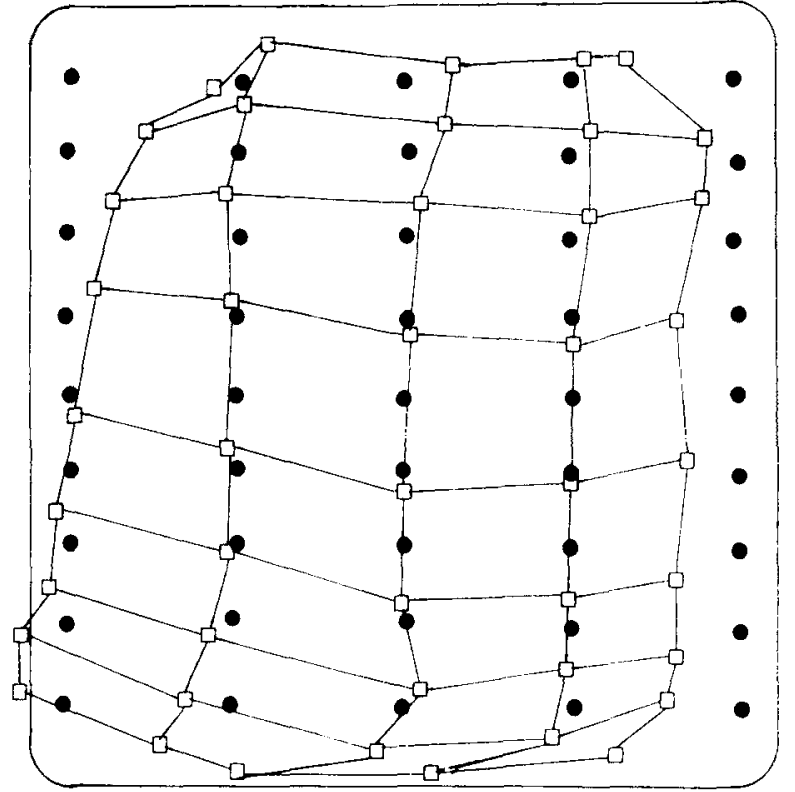

Figure 2. Display demonstrating the relation between point of gaze and the eye monitor's uncalibrated readings. Closed circles represent actual eye point-of-gaze; open squares represent eye monitor's uncalibrated readings.

account for: translation, magnification, and linear "crosstalk" from the opposite eye. The following equations define the transformations: $x^{\prime}=f(x, y)=$ $\mathrm{ax}+\mathrm{by}+\mathrm{c}, \mathrm{y}^{\prime}=\mathrm{g}(\mathrm{x}, \mathrm{y})=\mathrm{dx}+\mathrm{ey}+\mathrm{f}$, where $\mathrm{x}$ and $y$ are the horizontal and vertical inputs from the eye monitor, where $x^{\prime}$ and $y^{\prime}$ are the true coordinates of the eye point-of-gaze on the CRT screen, and where $a, b, c, d, e$, and $f$ are coefficients to be set by the calibration process.

The calibration program operates by accepting the $x$ and $y$ coordinates for three points from the experimenter via the Teletype. The program then displays these points one at a time. When the subject has fixated on the point being displayed, he signals this by pressing a push button. The program then repeatedly reads the $\mathrm{A} / \mathrm{D}$ converter and takes an average of these values. The number of reads taken and the time between reads in milliseconds are parameters which are set by the experimenter. The program repeats this procedure for the other two points and uses the input readings obtained to compute the coefficients of the two transformation equations. The transformation equations are applied to all subsequent in puts or until another calibration is done.

Verification. Another routine exists which verifies that the calibration program produced a valid set of transformation functions. The program displays one point on the CR'T, reads the eye monitor's outputs a number of times, applies the transformations to these input values, and then displays the original point along with the transformed points. The calibration program maty be repeated until the verification progran shows that the desired degree of accuracy has been reached. For this program, the experimenter controls the following parameters: the coordinates of the initial point, the number of reads to be made, and the number of milliseconds between reads.

Through the use of these programs and other off-line tests, it was discovered that the correspondence between the eye monitor's readings and the actual eye point-of-gaze is not a linear function. Rather, it is only linear for a subset of the area subtended by the CRT screen, namely the area of the center (see Figure 2). The area over which the transformation is linear varies from subject to subject and is very sensitive to changes in the position of the sensors relative to the eyes. Another program, described next, was written to provide more information about the actual relationship between the eye monitor's outputs and the subject's point-of-gaze.

Monitoring. This program displays a $9 \times 9$ grid of points and a tracking cross which moves as a function of the eye monitor's outputs. The grid covers the entire screen, and the points are thus $3.025 \mathrm{~cm}$ apart. The experimenter can elect for the cross to move according to the actual (raw) input voltages from the monitor or according to the calibrated monitor inputs. The experimenter also determines the number of reads the program is to make before taking an average. Averaging is used here to make the cross appear more stable by smoothing the effects of eye tremors. The experimenter may elect to monitor the horizontal sensor separately, the vertical sensor separately, or both dimensions together. The tracking cross may also be present, absent, or present at the edge of the screen where the subject cannot see it. Without the tracking cross, the experimenter can monitor, one at a time, the vertical and horizontal sensors using the meter on the eye monitor's control box; however, this method is slower and less precise.

Prior to an experiment, the experimenter would use this program to aid him in adjusting the sensors' positions and the monitor's control knobs so as to maxinize the linearity of the eye monitoring. The monitor control box has knobs for each eye which change the relative zero position of the sensor's voltage, the magnititication of the output signal and the anount ol crosstalk from the opposite sensor. By adjustirg these knobs appropriately, using the tracking cross as a guide, the experimenter can perform a linear calibration electronically and thereby accomplish the same result as running the calibration program.

Through the use of this program, it has been discovered that the area over which the monitoring is linear subtends a horizontally oriented rectangle. It has never been possible to get linearity over the entire area covered by the CRT screen, but for most subjects, one can adjust the monitoring so that it is lineall over that portion of the screen which is used in 
this experiment. The monitoring program was the tirst one run during an experimental session. It made possible the elimination, early in the experiment. of those few subjects for which no linear calibration could be obtained.

Pre-/posttests. For purposes of the experiment to be reported below, programs concerned with a pretest. a simulation period, and a posttest were also written. The pre-and posttests are basically identical. Their purpose is to measure and compare the subject's perception of straightness immediately before and after the simulation period. The pre-/ posttest program displays a curved dotted line which changes shape. (A dotted line was used because a truly curved line cannot be plotted with this display.) The curve is first shown as a parabola opening either up or down. The end points remain fixed, while the curvature becomes progressively shallower. The parabola eventually passes through a straight line and then begins to curve the opposite way. The parabola continues to bend up and down until the subject stops it. The subject controls the display through the push buttons. One button stops the movement, another button starts it again, while a third and fourth button change the curvature a single increment at a time, up or down. A fifth button is pressed when the subject perceives the line to be straight. The program records this curvature. prints it on the Teletype, and punches it on paper tape. The number of pretests (posttests) that are to be run is a program parameter. The starting curvatures of each successive pretest or posttest are equal in magnitude, but alternate up and down to prevent bias.

Other parameters are: the initial curvature of the parabola, the distance between dots, and the vertical position of the parabola on the CRT. The program calculates the subject's perception of straightness by taking the average of his pretest settings. The experimenter may choose to use the true value of straightness during the following simulation program or he may choose to use the subject's perception of straightness.

Upon completion of the pretest program, there is a brief time-out. after which the simulation program is automatically begun.

Simulation. For the simulation period, the experimenter selects an image and a transformation. The transformation simulates the prism and is a function of the position of the subject's point-of-gaze. The progran displays the transformed image for a specified period of time while continually monitoring the subject's eye movements and distorting the image according to these movements. In this experiment, 10 readings of the eye monitor are taken and averaged, resulting in one eye position being calculated approximately every $6 \mathrm{msec}$. At this rate, one cannot perceive any lag between the display and one's eye movements.
The image is composed of dots and can currently be chosen from among the set of horizontally oriented parabolas. The experimenter selects "C." the curvature, and "E," the $y$ coordinate of the end points. which will be used in the equation: $\mathrm{Y}=$ $C^{*} X^{*}(1023-X)+E$. He also selects the distance between dots which omprise the image.

The transformation is a second-degree equation of the form: $Y=A^{*}\left(X_{f}-X\right)^{2}+Y_{o b j}$. " A " is the magnitude of the curvature of the transformation which represents the power of the hypothetical prism. $X_{f}$ is the $x$ coordinate of the subject's point-of-gaze. $Y_{o b j}$ is the $y$ coordinate of the image. This type of transformation is a simplification of the actual transformation caused by a horizontally oriented wedge prism, but it preserves the features which are most striking. Under this transformation, a horizontal lines becomes a parabola. which opens either up or down. The transtormed image will always be tangent to the actual image at the $x$ coordinate of the subject's point-of-gaze. If the image is a parabola of curvature " $K$," then a transformation of curvature " $\mathrm{K}$ " will result in the display of a straight line that tilts back and forth according to the subject's horizontal eye movements. As the subject scans this "straight line." in order to maintain fixation, he must move his eyes in a curved path. In the other case, where the subject sees the curved transformation of a straight-line image, he must move his eyes in a straight path in order to keep the "curved" object tixated.

As an option. the simulation program also displays monitoring information at the top and bottom edges of the screen, concealed from the view of the subject by the cardboard box that surrounds his head. This monitoring informs the experimenter about how the subject is moving his eyes. If he is not scanning the image so as to keep it fixated, the experimenter may want to provide further instructions. The monitoring can also be used to determine if the calibration transfornation has changed. This may be the result of head movements, squinting of the eyes from fatigue, etc. The experimenter can make some calibration adjustments by changing the control knobs on the eye-monitoring box during a rest period when the subject has his eyes closed. The monitoring information includes dots at the bottom and top-right of the screen which move according to horizontal and vertical eye movements, respectively. An error message is also displayed at the bottom when the subject doesn't scan the image so as to keep it fixated. In the experiment to be described, the subject is supposed to move his eyes in a curved path. When setting the program parameters, the experimenter selects a vertical error criterion, "Y," which has the following meaning: "While scanning from the center to either edge of the screen or from the edges back to the center, the subject must move his eyes vertically at 
least ' $Y$ ' raster points." The program displays an " $\mathrm{X}$ " for a few seconds at the horizontal position where the subject violated this criterion, either at the left edge. the center, or the right edge. There is also the option of ringing the Teletype bell upon error to inform the subject of his mistakes. In the upper left edge of the screen are displayed two lines. The distance between the lines represents the vertical distance which the eye must move for a successful scan. A dot is displayed in relation to these lines showing the actual vertical movement for the previous scan.

The sinulation session is divided into alternate simulation periods and rest periods. The number of simulation periods, their length (in seconds), and the length of the rest periods (in seconds) are all program parameters.

At the end of each simulation period, the period number, the number of correct and incorrect scans, and the order in which they occurred are output on the Teletype and paper-tape punch.

\section{THE EXPERIMENT}

\section{Procedure}

Nine subjects were used in this experiment. They were selected at randon from a pool of college student volunteers, the only requirenent being that they not wear glasses or contact lenses. The experiment was designed to simulate exposure to an object curved downward which was transtormed by a prism to appear straight. The apex of the parabolic object was at the center of the CRT and its end points were 64 raster points $(1.512 \mathrm{~cm})$ below the center at the left and right edges of the screen. The curvature of the image was chosen so as to require approximately the same amount of vertical cye movement during scanning as in previous experiments employing contact lens prisms. Since the CRT screen altowed less horizontal range than did the apparatus in the previous experiments. the actual degree of curvature in this experiment was greater. The dots in the stimulus, pretest, and posttest images were 26 raster points, or about $.014 \mathrm{~cm}$ apart.

The headrest was positioned so that the subject's eyes would be $53 \mathrm{~cm}$ from the CRT and his line of sight would be approximately perpendieular to the sereen of the CRT at its center. The aperture in the cardboard box permitted viewing. with both eyes, of the entire width of the (RT screen (about $26 \mathrm{deg}$ ) but only enough of the height to render the stimulus visible at all times.

At the beginning of the experiment. the experimenter adjusted the variable pretest line so as to look straight through an actual glass prism (30 diopter. base down). The subject was told that the experiment involved prismatic distortion. The general character of such distortion was described to him and demonstrated with the prism. He held the prism up to one eye and noted the straightening effect. He then moved his head from side to side (moving the prism along with it) and noted the tilting of the image. (The transformation resulting from head movement is the same as that resulting from eye movement with a contact lens prism.) It was emphasized that the actual line on the screen did not move. He was then told that the experiment would involve similar effects resulting from a prism and that his eye movements would be monitored. He was not told that the experiment was, in fact, a simulation and that his eye movenent would actually be producing the transtormations.

The headrest was then put in position and the cardboard box removed. The subject was told that the prism to be used in the experiment was mounted in the box. The monitoring program was then started with the eye-movement monitor turned off. This positioned the cross in the center of the screen, where it served as the target to be centered in the slit during head positioning. The headband was fitted and set aside. Then the adjustment of head position was carried out as described above. Next, the pre-/posttest program was called in, so that the operation of the push buttons could be explained and demonstrated. The subject was given two or three practice trials to get used to operating the buttons without looking at his hatrd.

At this point. the course of the experiment (to be described bekw) was outlined to the subject. Finally, the headband, with the senorrs altached. was positioned and calibration pertormed. In this experiment. unly horizontal movement (involving the sensors positioned betore the right eye) was calibrated. since only such movements afficted the transformation. Furthermore, the calibration area included only the three center rows of the $9 \times 9$ grid, as the sinulated object lay entirely within this area. These restrictions made the linearity problem as discussed previously less severe. Only the monitoring program with raw input voltages was used for ealibration. The experimenter indicated grid points to be fixated. and adjusted the sensors and controls so that the cross approximately matched these points in the horizontal dimension. No rigid quanntative criteria were employed. Calibration was considered satisfactory it, during two or three point-by-point scans of the three center rows the cross was, for the most part, closer to the stccessise fixation points than to any others.

The experiment was run with the room lights out and the cardboard box mplaced. The experimenter had some light from the computer. but the subiect could see nothing but the image on the weren. The wbjet was told that the prism was not installed. The pretest program was run to obtain eight measures of straightness. While viewing the pretest image, the subject was instructed to try to fixate the center at all times. even it this seemed unnatural or ditficult. The tirst four of the eight settings were treated as practice. The lase lour cinstituled the pretest data, and the average of these was used by the program to modify the stimulus in the simulation period. After the pretest settings, while the subject had his eyes closed. he was lold that the prism was being installed. When he opened his eys. the simulation program was running. He was now toki to scan the line systematically back and forth. In going from one end w the wher, he was instructed to fixate successively five regions of the line: the left end, a point midway between the left end and the conter "lett center"), the center, the "right center." and the right end. It was emphasized that he should always try to tixate the line dexplte its apparent movenent. He continued to scan until the beginnitig it a rest period. at which point he was to close his eyes. The heginning of a rest period was signaled by the disappearance of the stimulus. At the end of a rest period, before resuming scanning. the subject was told to fixate indicated regions so that calitsation could be checked

Alter the eight pretests, the experiment consisted of three successive identical sections. Each section consisted of a simulation period followed by eight posttests. The simulation period was further divided into three training sessions of 4 min each, which were separated by 1 -min rest periods. There were thus three different stets of posttests. reflecting different cumulative degrees of prism exposure.

After the experiment, the subjects were told that a simulation had been involved and were asked questions designed to determine whether they had suspected this: "Did you believe that a prism was present?" "Did you believe that the line was stationary on the srect." They were also asked to describe or draw the approximate shape of the path they thought their eyes had been making during scanning (c.g.. straight. curved upward, or curved downward).

The prediction, derived from the theory as stated by Festinger et al. $(1967)$ and Slotnick (1969), was that as a result of this training. postlest settings of straightness should manitest a tendency to have a lower y value at the center of the screen than was characteristic of the settings made during the pretest. The argument behind this prediction goes as follows: If the subjects find during training that what appears to be straight can only be tixated by a downwardly curved eye path, then a truly straight eye path will onls fixalte a contour that appears curved up. Hence, judgments of 
straightness during the posttest should be more curved up (i.e., have a lower $y$ value) than those given during the pretest.

\section{Results}

Before presenting the results concerning the prediction, another finding must be mentioned. The eight posttests given after each of the three sections turned out not to be homogeneous, with the first four scores averaging out more negative (i.e., more adaptive) than the last four scores in all three sections and, as a result, overall. That is, the adaptation effects produced by the training appeared to be negated quickly by the normal visual experience engendered with the posttest. The mean differences in raster units between the first four and the last four posttest measures were $2.19,3.31$, and 0.86 , respectively. The second of these and the average of all three were significant: $\mathrm{t}=3.62, \mathrm{p}<.01$, and $\mathrm{t}=$ $4.03, p<.01$, respectively.

Because of these differences between the first four and last four posttest scores, the posttest was divided into two parts, and adaptation scores (average pretest scores minus average posttest scores) were computed both for the first four and the last four posttest scores. In the case of the former, the means for the posttest were, as predicted, lower than the means for the pretest in all three sections. The mean differences in raster units (pretest minus posttest) are, respectively, $3.39,4.33$, and 1.06 . The differences were significant for Sections 1 and 2 and for all sections combined, $t$ $=2.88, \mathrm{p}=.02 ; \mathrm{t}=4.82, \mathrm{p}=.001 ;$ and $\mathrm{t}=3.38, \mathrm{p}$ $=.01$, respectively. None of the differences between the last four posttest scores and the pretest were statistically significant, though, except for Section 3 scores, they were all in the predicted direction. The fact that the third section scores were generally not significantly adaptive may be accounted for by comments which were made by at least some subjects about having experienced more fatigue during the third section than during the others and having experienced actual difficulty in fixating the apparently moving line during that section. Some subjects suggested that at times they had, in fact, been unable to fixate it.

In general, the above results warrant the conclusion that the simulation technique that has been created does produce visual rearrangements which are responded to by subjects in a manner highly similar to those produced by prisms. In fact, the experiment reported provides a statistically secure validation for the findings reported by Festinger et al. (1967) and Slotnick (1969).

One of the main areas of further work will be an attenipt to use the system in conditions in which both the $x$ and $y$ dimensions of eye movement are required, rather than only the $x$ dimension as at present. This would make possible the study of changes in eye movement occurring during training. Even with the present, more restricted, system, however, a large number of further studies can be done-for example, studies that vary the type of training given subjects. In a study already pilot tested by the authors, visual rearrangements are introduced, not, as in past research, by a single prism of given orientation and power, but by many prisms of different degrees of power and orientation. This makes it impossible for subjects to connect any new perception of form to a given retinal projection or sign. Instead, it forces them to concentrate exclusively on learning a new rule about an efferent-reafferent relation. In another planned study, the inhibiting effect due to old reafferent signs will be reduced by impoverishing the visual input seen by subjects during training. Instead of seeing straight lines or curves, each of which recall prior perceptual experience, moving as a function of their eye movements, subjects will see only one or a few points moving when they move their eyes. These types of studies attempt to study the adaptation process in finer detail than has been done hitherto. The result may be a greater knowledge of the conditions that enhance or inhibit adaptation. The tlexibility afforded by the simulated system presented in this paper will be particularly helpful.

\section{REFERENCES}

Festinger, L., Burnham, C. A., Ono, H., \& Bamber, D. Efference and the conscious experience of perception. Journal of Experimental Psychology Monograph, 1967, 74(4, Whole No. 637).

GYR, J. W. Is a theory of direct visual perception adequate? Psychological Bulletin, 1972, 77, 246-261.

Gyr, J. W., Willey, R., \& Gordon, D. G. Correlations between motor learning and visual and arm adaptation under conditions of computer simulated visual distortion. Perceptual and Motor Skills, 1972, 35, 551-561.

SLOTNICK, R. S. Adaptation to curvature distortion. Journal of Experimental Psychology, 1969, 81, 441-448.

\section{NOTES}

1. The idea of simulating a prism was originally suggested several years ago by Arthur Zivian, who was then working on the project. The idea was first put into operation for the simulation of prism goggles, rather than the contact lens-prisms combination (see Gyr. Willey, \& Gordon, 1972).

2. Biometrics Division/Narco Bio-Systems, Inc., P.O. Box 12511, 7651 Airport Boulevard, Houston, Texas 77017.

(Received for publication November 4, 1974; revision received December 22,1974 .) 